\title{
Perceptual Hash Image Classification Algorithm based on SIFT Feature
}

\author{
$\mathrm{Xi} \mathrm{Li}{ }^{1}$, Bin Tian*, ${ }^{*}$, Nan $\mathrm{Xu}^{1}$ \\ ${ }^{1}$ School of Electrical and Information Engineering, Wuhan Institute of Technology, \\ Wuhan, Hubei Province, 430205, China \\ *Corresponding author
}

Keywords: HOG; Least Second Norm; PCA; Image Classification; Image Feature

\begin{abstract}
Image classification with wide application in computer vision refers to an image processing method which classifies different categories of goals based on different features reflected in image information. BOW-SVM is a typical image classification method with higher accuracy but unsatisfactory operating performance. To improve performance and accuracy more effectively, an efficient image classification method based on HOG-PCA is presented. First, extract and whiten features of histogram of oriented gradients (HOG), then make down sampling randomly to unify scale, and then make feature mapping through principal component analysis (PCA), and finally make nearest neighbor classification through least second norm determination. $\mathrm{C}++$ is adopted in the experiment where extraction is made through OPENCV and Darwin and test is made in the PASCAL 2012 dataset, and the experiment compares accuracy and operating performance of this method and BOW-SVM, proving that the presented method is more efficient and of better operating performance.
\end{abstract}

\section{Introduction}

Image classification is a classification method which by making quantitative analysis on image through computer based on different features reflected by image information, divides image or image region or even each pixel point of image into certain category. Image classification has wide application in such areas as military for target identification of airplanes and missiles, industrial manufacture for product quality inspection, science for satellite cloud image and infrared image analysis, medicine for leukocyte identification and ultrasonic liver image identification and daily life for scene identification. However, for human and natural factors, there are still some problems in image classification. By now, image classification has become difficult and hot issue of research in image processing area [1].

Effective representation of image features is the basis to get good classification result. For that, lots of image representation methods have been presented, in which the following are relatively representative: Ojala and etc [2] presented Local Binary Pattern (LBP) for textural features, a description algorithm with strong robustness to coordination but lack of handling of scale and rotation. Lowe and etc [3] presented Scale Invariant Feature Transform (SURF), which could adapt to image scale, rotation and affine transformation and is wildly used in scene matching, target identification, face identification, image mosaic and etc. But it is time-consuming to compute regional features through this method. Bay and etc [3] presented Speed Up Robust Features (SURF) whose computing time precedes SIFT and which can adapt to scale transformation but lacks certain accuracy. In recent years, Histogram Of Gradient (HOG) presented by Dalal and etc has been widely used for its good adaptability to light, scale and direction.

\section{Image Classification}

A method based on BOW-SVM [6] is typical in image classification. BOW (Bag of Word, $\mathrm{BOW}$ ), originated from language and information processing area, is a histogram representation 
based on independent feature, taking image as document and feature as phrase when applied in computer vision area. It usually contains three procedures: feature extraction, feature description and feature phrase generation.

Classifier is needed in image classification based on BOW, and support vector machine (SVM) is a relatively typical one [8]. It maps data into a higher-dimensional space where maximum margin hyperplane is established. Two parallel hyperplanes are established on both sides of hyperplane separating data. Separate hyperplane to maximize distance between the two parallel hyperplanes. The classifier is effective in classification, but has no obvious advantages in training performance. In general, classification method based on BOW-SVM provides good performance, but it lacks consideration of spatial relationship between feature points, and it takes much to establish phrase and model training.

Therefore, we present a image classification method based on HOG-PCA. First, extract image features effectively by using good adaptability of HOG to light, scale and direction, and then whiten features to reduce abnormal points, and finally exploit computing performance advantage of EigenFace. The method is simple with good computing performance and adaptability to image category, and especially adapts to classification of natural image, which are its major advantages. And its basic framework is as Figure 1:

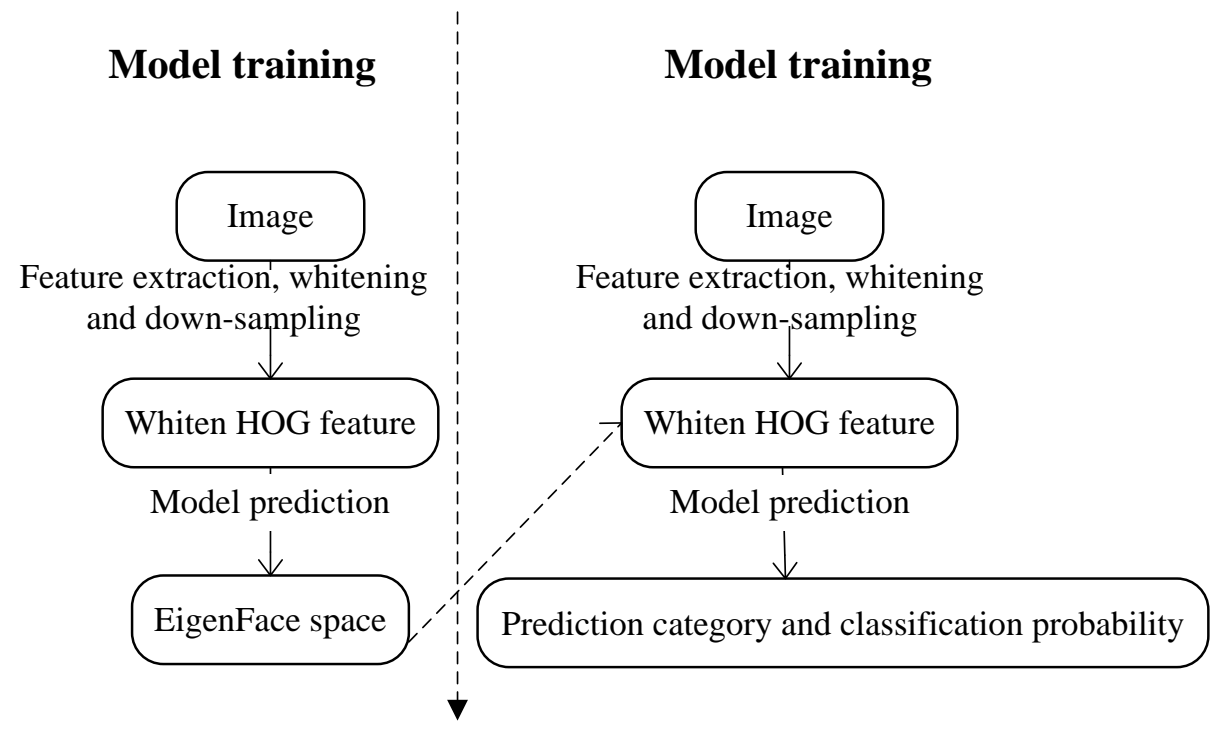

Fig.1 Image classification method based on HOG-PCA

The presented method is tested in dataset PASCAL 2012 [9] and is compared with BOW-SVM. The experiment proves that the method presented by us is better in accuracy and operating time.

\section{Image Classification Algorithm based on Sparse and Low-rank Matrix}

Three procedures are usually included in mage classification: image feature extraction and processing, classification model establishment and classification evaluation [10]. First, extract image features through HOG, then whiten features and make down sampling, and then establish PCA mapping model of training sample HOG features, and finally use model to classify through least square method.

\section{HOG feature extraction}

The core idea of HOG is that outline of tested partial object can be described by light intensity gradient or edge orientation distribution. There are five procedures to compute HOG:

1. Image gamma and color standardization

When evaluating color space, such as grayscale, RGB and LAB, it usually needs to be transformed into grey image for unified evaluation standard. And grey value shall be transformed to the range of 0 to 1.

2. One-dimensional gradient filtering computing direction

Usually the simplest method is most effective, and gradient computation method is very 
important for correct description of image feature. In HOG computation, Sobel operator is usually used to compute image difference of first-order $x$ or $y$ axis to get good performance. Sobel operator, together with Gaussian blur and difference, can make result possess better anti-noise performance.

3. Weighted voting of spatial and directional cells

It is the most important step. Set accumulated weight for direction of each cell. First, algorithm for directional value computation is:

$$
\begin{aligned}
& x(i)=\cos (\theta) \\
& y(i)=\sin (\theta) \\
& \theta=\theta+\pi /\left(N_{\text {direction }}+1\right)
\end{aligned}
$$

Where, $i$ is directional No., $\theta$ is angel and initial value is $0, x(i)$ is directional weight of difference of $i$ in $x$ axis while $y(i)$ is directional weight of difference of $i$ in $y$ axis.

Then compute amplitude and direction. Amplitude is mean square value of image difference of $x$ or $y$ axis. $N_{\text {direction }}$ is the total direction number, and usually set as 9 . Directional value is the maximum weighted value in each direction.

4. Establishing Block and standardization

Summarize features in cells into Block and its algorithm is:

$\mathrm{B}(\mathrm{x})=(\mathrm{C}(\mathrm{x})-\mathrm{B}($ size $)+1) / \mathrm{B}($ step $)$

Of which, $\mathrm{B}(\mathrm{x})$ represents amount of Block in $\mathrm{x}$ axis, $\mathrm{C}(\mathrm{x})$ represents amount of cells in $\mathrm{x}$ axis, $\mathrm{B}$ (size) is size of Block and $\mathrm{B}($ step) is the step of Block's change. And Block amount in $y$ axis can be computed in the similar way.

Standardization has two steps (1) Feature shear. Shear by setting range of minimum and maximum eigenvalue, eliminate smaller and larger values and unify again the eigenvalues of residue features to the scale of 0 to 1 ; (2) Feature standardization.

5. Establishing image HOG

Dimensionality reduction can reduce feature amount and computing complexity, thus, setting imputed dimension according to needs. Energetic and directional sum: sum eigenvalues of different directions and Blocks to form image HOG. Compared with other descriptors, descriptor got through HOG keeps invariability of geometric and optical transformation. An example of HOG feature is as follows.

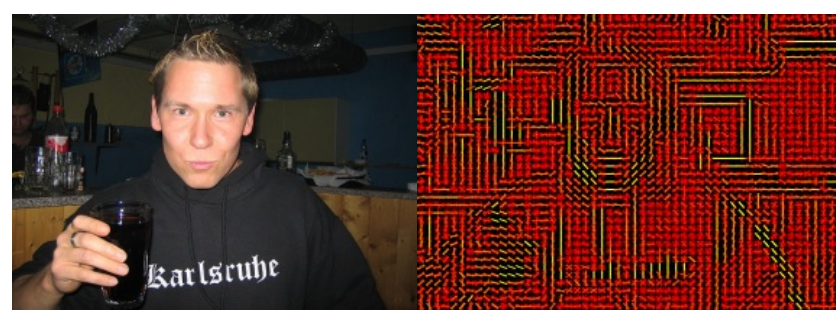

Fig.2. Image and its HOG feature

In Figure 2, image is on the left, and its colorful HOG feature is on the right.

\section{HOG feature whiting and down sampling}

For HOG feature still has high space coupling, HOG feature shall be whitened based on two purposes: (1) to reduce high association between features, (2) to make same variance for training feature. Feature whiting can be summarized as follows [11]:

Suppose $X, d$ dimension and $n$ line $(d \times n)$, to reduce association between features, transform it as follows

$$
Y=W X
$$

Of which, $Y$ is post-transformation feature, $W$ is whiten matrix, and to make $W$ eliminate feature association, $Y Y^{T}$ shall be diagonal matrix, namely

$$
Y Y^{T}=(n-1) I
$$

There are many $W$ which can meet formula 6 , thus making $W=W^{T}$, and then $W$ can be computed through formula 7

$$
W=\sqrt{n-1}\left(X X^{T}\right)^{-\frac{1}{2}}
$$


For inconsistent scale of image classified, down sampling or up sampling shall be made randomly. If whiten feature amount is more than required feature amount, make down sampling.

$Z=\operatorname{subsample}(Y, m)$

Of which, $Y$ is post-whitened feature, $Z$ is post-sampled feature, with total number of $m$, the function of subsample extracts features randomly by sampling.

\section{PCA mapping}

Principal Component Analysis (PCA) [12] reduces data dimension by restricting parameters with less information through K-L transformation, but takes much to compute covariance matrix. PCA transforms multi-dimensional image information into several principal components which involve most of image information, thus improving classification efficiency [13].

Suppose $N$ sample features $\left\{x_{1}, x_{2}, \ldots, x_{N}\right\}$, and each feature is a $n$-dimensional space and belongs to certain type of category $\left\{X_{1}, X_{2}, \ldots, X_{c}\right\}$. Certain linear transformation shall be considered, mapping from $n$-dimensional space to $m$-dimensional shall meet $m<n$, and new feature vector $y_{k} \in R^{m}$ can be used to define the linear transformation as follows:

$$
y_{n}=W^{T} x_{k} \quad k=1,2, \ldots, N
$$

Here, $W^{T}$ is a matrix with orthogonal columns. Suppose the definition of scatterplot matrix of the overall sample is as follows:

$$
S_{T}=\sum_{k=1}^{N}\left(x_{k}-\mu\right)\left(x_{k}-\mu\right)^{T}
$$

Of which, $N$ is sample size, and $\mu \in R^{n}$ is mean value of all samples. Then linear transformation can be used.

$$
\begin{aligned}
W_{\text {opt }} & =\arg \max _{W}\left|W^{T} S_{T} W\right| \\
& =\left[w_{1}, w_{2}, \ldots, w_{m}\right]
\end{aligned}
$$

Here, $\left\{w_{i} \mid i=1,2, \ldots, m\right\}$ is maximum feature vectors corresponded by $n$-dimensional feature space scatter set $S^{T}$, and it is also the mapping feature of model.

\section{Classification discrimination}

Compute second-order space between each sample and training sample to make out the classification of minimum distance and classify it as classification of this training sample.

1. Project image feature to be identified to PCA space.

2. Compute post-projecting feature and distance between training samples in PCA space.

$$
d(i)=\sqrt{\left(t_{1}(i)-p_{1}\right)^{2}+\left(t_{2}(i)-p_{2}\right)^{2}+\ldots+\left(t_{n}(i)-p_{n}\right)^{2}}
$$

Of which, $d(i)$ is the distance from $i$ th training samples, $t_{1}(i)$ is the first projecting eigenvalue of ith training samples, $p_{1}$ is the first projecting eigenvalue of certain testing sample. $t_{2}(i), p_{2}$, $t_{n}(i)$ and $p_{n}$ have similar meaning.

3. Finally, choose classification with minimum space as its classification.

4. Calculation method of classification reliability. Distance between the two is chosen to be the reliability.

\section{Conclusion}

The Paper analyzes general principles, steps and evaluation methods of image classification and presents a simple but efficient image classification method. For inherent advantages of HOG feature, the method takes account of image rotation, coordination, scale and etc, and is able to make fast classification of image.

In experiment, $\mathrm{C}++$ is used to carry out the presented method which is more efficient than common Matlab simulation method, and is more advantageous in operating performance, total accuracy, average precision and average recall then traditional BOW-SVM.

Image classification has wide application value, and its classification accuracy also depends on further analysis and research on more complex factors on the image such as scene, color, shadow and others, which has always been the key and difficult point of image classification research, and 
is also one of the important development directions of image classification.

\section{Acknowledgement}

Scientific research project of Hubei provincial education department in 2016, No.B2016061; Scientific research foundation of Wuhan Institute of Technology in 2015, No.K201501

\section{References}

[1] Lv, Z., Tek, A., Da Silva, F., Empereur-Mot, C., Chavent, M., \& Baaden, M. (2013). Game on, science-how video game technology may help biologists tackle visualization challenges. PloS one, 8(3), e57990.

[2] Lv, Z., Halawani, A., Feng, S., Li, H., \& Réhman, S. U. (2014). Multimodal hand and foot gesture interaction for handheld devices. ACM Transactions on Multimedia Computing, Communications, and Applications (TOMM), 11(1s), 10.

[3] Lv, Z., Halawani, A., Feng, S., Ur Réhman, S., \& Li, H. (2015). Touch-less interactive augmented reality game on vision-based wearable device. Personal and Ubiquitous Computing, 19(3-4), 551-567.

[4] Lv, Z., Li, X., Zhang, B., Wang, W., Zhu, Y., Hu, J., \& Feng, S. (2016). Managing big city information based on WebVRGIS. IEEE Access, 4, 407-415.

[5] Zhang, Z., Ou, J., Li, D., \& Zhang, S. (2017). Optimization Design of Coupling Beam Metal Damper in Shear Wall Structures. Applied Sciences, 7(2), 137.

[6] Shen, W., Li, D., Zhang, S., \& Ou, J. (2017). Analysis of wave motion in one-dimensional structures through fast-Fourier-transform-based wavelet finite element method. Journal of Sound and Vibration, 400, 369-386. 